\title{
Resting metabolic rate and energetics of reproduction in lactating Eothenomys miletus from Hengduan mountain region
}

\author{
Wan-long Zhu* and Zheng-kun Wang
}

\begin{abstract}
Background: It has been advocated that variation in resting metabolic rate (RMR) may affect the reproductive performance of female animals. In order to investigate the relationships between RMR and reproductive output in lactating Eothenomys miletus, body mass, RMR, food intake, litter size and mass, as well as the weight of visceral organs and gastrointestinal tract were measured in the female E. miletus prior to reproduction and at late lactation.

Results: It showed that RMR was 39.62\% higher at late lactation than prior to reproduction. There was no significant correlation between RMR prior to reproduction and reproductive output. However, RMR at late lactation was positively correlated with body mass, food intake, litter size and mass, and weight of visceral organs and gastrointestinal tract at late lactation, within which RMR was more related to gastrointestinal tracts than the visceral organs. Moreover, serum leptin levels were positively correlated with body fat mass, RMR, and food intake at late lactation.
\end{abstract}

Conclusions: Our data supported the hypothesis that animals with higher RMR during lactation may have a greater digestion and absorption capacity in the digestive system for absorbing energy and may be able to devote more energy for reproduction. Leptin may participate in the regulation of body mass in lactating E. miletus.

Keywords: Eothenomys miletus; Resting metabolic rate; Serum leptin levels; Lactation

\section{Background}

Sustained energy intake (SusEI) has significance in defining upper energetic limits to the ability of distribution, survival, and reproduction in mammals (Zhao 2010). Previous studies demonstrated that SusEI was constrained intrinsically by some aspects of physiological processes (Speakman 2000; Speakman and Król 2005, 2010). For example, limitation of SusEI was imposed by the expenditure capacities of the energy-consuming organs, such as the mammary glands during lactation (Zhao et al. 2010a). Lactation is the most energetically demanding period in the life cycle of female mammals. Female animals appeared to approach an upper energetic limitation of SusEI during lactation (Zhao et al. 2010b). It has been reported that mammals with higher resting metabolic rate (RMR) during lactation often had lower reproductive output at interspecific levels (Thompson et al. 1986). But other

\footnotetext{
*Correspondence: zwl_8307@163.com

School of Life Science of Yunnan Normal University, Kunming 650500, China
}

researches showed that there was no correlation between RMR and reproductive output, especially in closely related species (Harvey et al. 1991). The advantage of researches on the relationship between RMR and reproductive output at interspecific levels was that there are relatively large scales of RMR, body mass, and life history characteristics in different species, but they may complicate the relationship between RMR and reproductive output because of the different genetic backgrounds and habitats of those species (Johnson et al. 2001). In contrast, researches at intraspecific levels could reduce genetic background and habitat difference, which could help to clarify the relationship between RMR and reproductive output (Zhao et al. 2010c). Leptin is a $16-\mathrm{kDa}$ protein that is synthesized in the adipose tissue and secreted into the bloodstream. It is also hypothesized that leptin contributes to maintaining body mass by regulating food intake and energy expenditure (Friedman and Halaas 1998). Furthermore, the positive correlation between serum leptin levels and body fat mass has been found in many small mammals including 
Apodemus chevrieri (Zhu et al. 2011a) and Apodemus draco (Zhu et al. 2013a). Lactation also had significant effects on serum leptin levels in some small mammals (Aoki et al. 1999; Seeber et al. 2002).

To maintain long-term maximum SusEI, there is a need to increase the capacity of digestion and absorption in the digestive system while increasing energy consumption in the digestive system, leading to the increasing of RMR (Hammond and Diamond 1992). Many studies showed that the correlation of SusEI and RMR is mainly affected by the mass of the gastrointestinal tract (Garton et al. 1994). But other researches suggested that this relationship was not affected by the mass of the digestive tract (Selman et al. 2001). Therefore, the relationship between RMR and digestive tract function is still not clear.

Eothenomys is a proper genus in China, and animals of this genus were typical animals in Hengduan mountain region. Eothenomys miletus is an inherent species in Hengduan mountain region (Zhu et al. 2011b). In order to investigate the relationships between RMR, reproductive output, and visceral organs in lactating E. miletus, body mass, RMR, food intake, litter size and mass, as well as the weight of visceral organs and gastrointestinal tract were measured in the female E. miletus prior to reproduction and at late lactation. We predicted that RMR prior to reproduction is positively related to reproductive output at the intraspecific levels, RMR at late lactation is positively related to reproductive output and visceral organs, and higher RMR during lactation is beneficial to enhance digestion and absorption capacity of the digestive system and also to increase energy intake for reproductive output in E. miletus.

\section{Methods}

\section{Samples}

E. miletus were obtained from a laboratory colony, which were captured in a farmland $\left(26^{\circ} 15^{\prime} \sim 26^{\circ} 45^{\prime} \mathrm{N}\right.$, $99^{\circ} 40^{\prime} \sim 99^{\circ} 55^{\prime}$ E; altitude 2,590 m) in Jianchuan County, Yunnan province, in 2010. E. miletus were maintained at a room temperature of $25^{\circ} \mathrm{C} \pm 1^{\circ} \mathrm{C}$, under a photoperiod of $12 \mathrm{~L} / 12 \mathrm{D}$ (with lights on at $0800 \mathrm{~h}$ ). Food (standard rabbit pellet chow; produced by Kunming Medical University, Kunming) and water were provided ad libitum. Twenty virgin female E. miletus aged 120 days were paired with males for 11 days in the summer of 2012, after which the males were removed. Nine of the females became pregnant, and their pups were weaned on day 22 of lactation (Zhu et al. 2013b). Body mass of females was weighed on days 3 and 22 of lactation, during which food intake of females was also measured. Food intake was calculated as the mass of food missing from the hopper, subtracting orts mixed in the bedding. Additionally, litter size and mass were recorded on days 3 and 22 of lactation. RMR was measured before mating (expressed as RMR prior to reproduction (Pri RMR)) and on day 22 of lactation (expressed as RMR at late lactation (LL RMR)). After day 22 of lactation, all females were sacrificed between $0900 \mathrm{~h}$ and $1100 \mathrm{~h}$ by decapitation. Blood was centrifuged at 4,000 rpm for $30 \mathrm{~min}$ after a $30-\mathrm{min}$ interval. Blood serum was collected and stored at $-75^{\circ} \mathrm{C}$ for leptin determination. All animal procedures were compliance with the Animal Care and Use Committee of School of Life Science, Yunnan Normal University. This study was approved by the Committee (13-0901-011).

\section{Measurement of resting metabolic rates}

Resting metabolic rates were measured by using an $\mathrm{AD}$ ML870 open respirometer (AD Instruments, Sydney, Australia) at $25^{\circ} \mathrm{C}$ within the thermal neutral zone (TNZ) (Zhu et al. 2012), and gas analysis was performed using a ML206 gas analysis instrument. Temperature was controlled by a SPX-300 artificial climatic incubator $\left( \pm 0.5^{\circ} \mathrm{C}\right)$ (Boxun company, Shanghai, China). The metabolic chamber volume was $500 \mathrm{ml}$, and airflow rate was $200 \mathrm{ml} / \mathrm{min}$. E. miletus were stabilized in the metabolic chamber for at least 60 min prior to the RMR measurement, and oxygen consumption was recorded for at least $120 \mathrm{~min}$ at 1-min intervals. Ten stable consecutive lowest readings were taken to calculate RMR (Zhu et al. 2013a). The method used for calculating the metabolic rate is detailed in Hills (1972).

\section{Morphology}

After collecting trunk blood in day 22 of lactation, the visceral organs, including the liver, brown adipose tissue (BAT), heart, lung, kidneys, spleen, and gastrointestinal tract (stomach, small intestine, cecum, large intestine), were extracted and weighed $( \pm 1 \mathrm{mg})$. Total body fat was extracted from the dried carcass by ether extraction in a Soxhlet apparatus (Zhang and Wang 2007).

\section{Measurement of serum leptin levels}

Serum leptin levels were determined by radioimmunoassay (RIA) with the ${ }^{125}$ I Multi-species Kit (Cat. No. XL85K, Linco Research Inc., St. Charles, MO, USA). The lowest level of leptin that can be detected by this assay was $1.0 \mathrm{ng} / \mathrm{ml}$ when using a $100-\mu \mathrm{l}$ sample size. The inter- and intra-assay variability for leptin RIA was $<3.6 \%$ and $8.7 \%$, respectively.

\section{Statistical analysis}

Data were analyzed using the software package SPSS 15.0. Prior to all statistical analyses, data were examined for assumptions of normality and homogeneity of variance using Kolmogorov-Smirnov and Levene tests, respectively. Body mass, RMR, food intake, and litter size and mass between day 3 and day 22 of lactation were analyzed using independent samples $t$ test. To detect 
possible associations of serum leptin levels with body fat mass, RMR, and food intake in the late lactation, we used Pearson correlation analysis. Pearson correlation analysis was also used to detect the relationship between RMR and body mass, food intake, and litter size and mass. Correlations between RMR and visceral organs were examined using partial correlation, with body mass as a covariate. Results are presented as means \pm SEM, and $P<0.05$ was considered to be statistically significant.

\section{Results}

\section{Body mass and RMR}

Body mass was $40.03 \pm 1.13 \mathrm{~g}$ and $26.92 \pm 0.69 \mathrm{~g}$ in day 3 of lactation and day 22 of lactation, respectively $(t=$ 8.54, $P<0.01$, Table 1 ). RMR prior to reproduction (Pri RMR) and RMR at late lactation (LL RMR) were 84.09 \pm $2.41 \mathrm{ml} \mathrm{O}_{2} / \mathrm{h}$ and $117.40 \pm 2.76 \mathrm{ml} \mathrm{O}_{2} / \mathrm{h}$. LL RMR was $39.62 \%$ higher than Pri RMR $(t=-9.08, P<0.01)$. Body mass was positively correlated with Pri RMR and LL RMR in lactating voles (Pri RMR: $r=0.823, P<0.01$; LL RMR: $r=0.794, P<0.01$, Figure 1$)$. But there was no relationship between Pri RMR and LL RMR ( $r=0.625$, $P>0.05$, Figure 2).

\section{Food intake and RMR}

Food intake was $5.45 \pm 0.16 \mathrm{~g}$ and $11.49 \pm 0.35 \mathrm{~g}$ in day 3 of lactation and day 22 of lactation, respectively $(t=-15.70$, $P<0.01$, Table 1). There was a positive correlation between food intake and Pri RMR and LL RMR in lactating E. miletus (Pri RMR: $r=0.677, P<0.05$; LL RMR: $r=0.780, P<0.05$, Figure 3$)$.

\section{Serum leptin levels and RMR}

Serum leptin level was $1.26 \pm 0.08 \mathrm{ng} / \mathrm{ml}$ in day 22 of lactation, and there was positive correlation between serum leptin levels and body fat mass $(r=0.777, P<0.05$, Figure 4A), LL RMR ( $r=0.762, P<0.05$, Figure 4B), and food intake $(r=0.670, P<0.05$, Figure $4 C)$ in lactating E. miletus.

\section{Litter size, litter mass, visceral organs, and RMR}

There was no significant difference of litter size between day 3 of lactation and day 22 of lactation $(t=1.033, P>$ 0.05 , Table 1$)$. But litter mass showed significant difference between day 3 of lactation and day 22 of lactation

Table 1 Body mass, food intake, litter size and litter mass during lactation Eothenomys miletus

\begin{tabular}{lllll}
\hline Parameters & Day 3 & Day 22 & $\boldsymbol{t}$ & $\boldsymbol{P}$ \\
\hline Body mass (g) & $40.03 \pm 1.13$ & $26.92 \pm 0.69$ & 8.54 & $<0.01$ \\
Food intake (g/day) & $5.45 \pm 0.16$ & $11.49 \pm 0.35$ & -15.70 & $<0.01$ \\
Litter size & $3.00 \pm 0.52$ & $2.33 \pm 0.37$ & 1.033 & $>0.05$ \\
Litter mass (g) & $3.94 \pm 0.14$ & $13.66 \pm 0.39$ & -23.43 & $<0.01$ \\
\hline
\end{tabular}

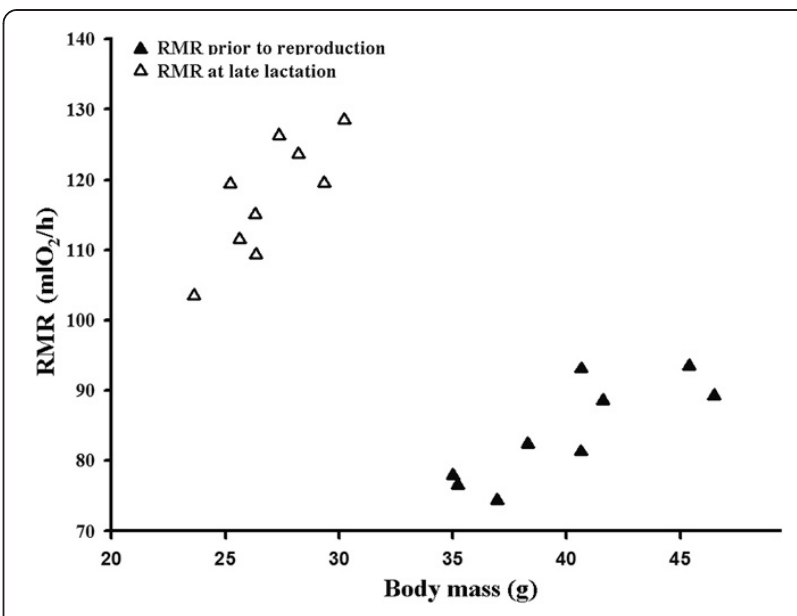

Figure 1 Relationships between resting metabolic rate (RMR) and body mass in lactating Eothenomys miletus.

( $t=-23.43, P<0.01$, Table 1$)$. No relationships were found between Pri RMR and litter size of early lactation (early L: $r=0.382, P>0.05$, Figure 5A) and litter size of late lactation (late L: $r=0.128, P>0.05$, Figure 5A). But LL RMR was positive with litter size of early lactation ( $r=0.697, P<0.05$, Figure $5 \mathrm{~B}$ ) and litter size of late lactation $(r=0.788, P<0.05$, Figure $5 \mathrm{~B})$. Similar to litter size, no relationships were found between Pri RMR and litter mass of early lactation $(r=0.363, P>0.05$, Figure $5 \mathrm{C})$ and litter size of late lactation $(r=0.356$, $P>0.05$, Figure 5C). But LL RMR was positive with litter mass of early lactation $(r=0.836, P<0.01$, Figure $5 \mathrm{D})$ and litter mass of late lactation ( $r=0.891, P<0.01$, Figure 5D). LL RMR was positively correlated with weight of visceral organs and gastrointestinal tract, within which the correlation between LL RMR and gastrointestinal tract was more related to other visceral organs (Table 2).

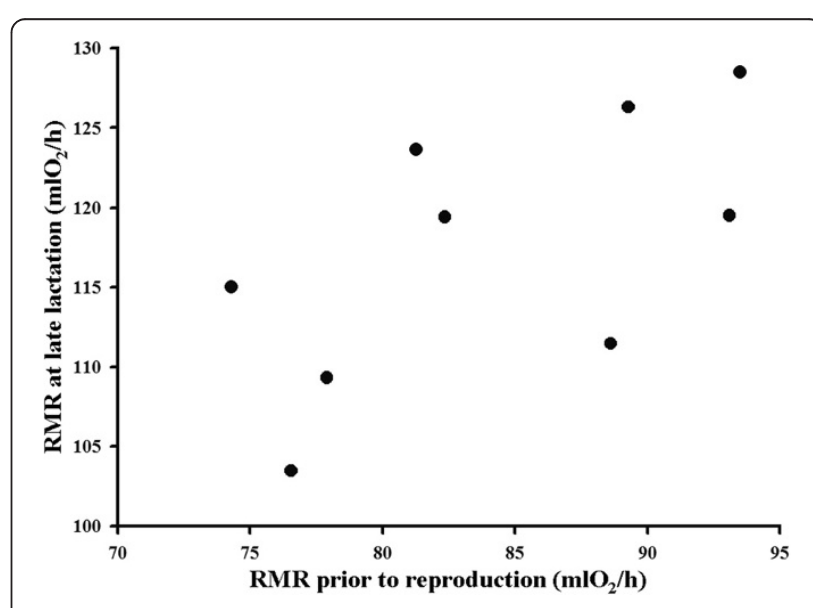

Figure 2 Relationships between RMR prior to reproduction and RMR at late lactation in lactating Eothenomys miletus. 


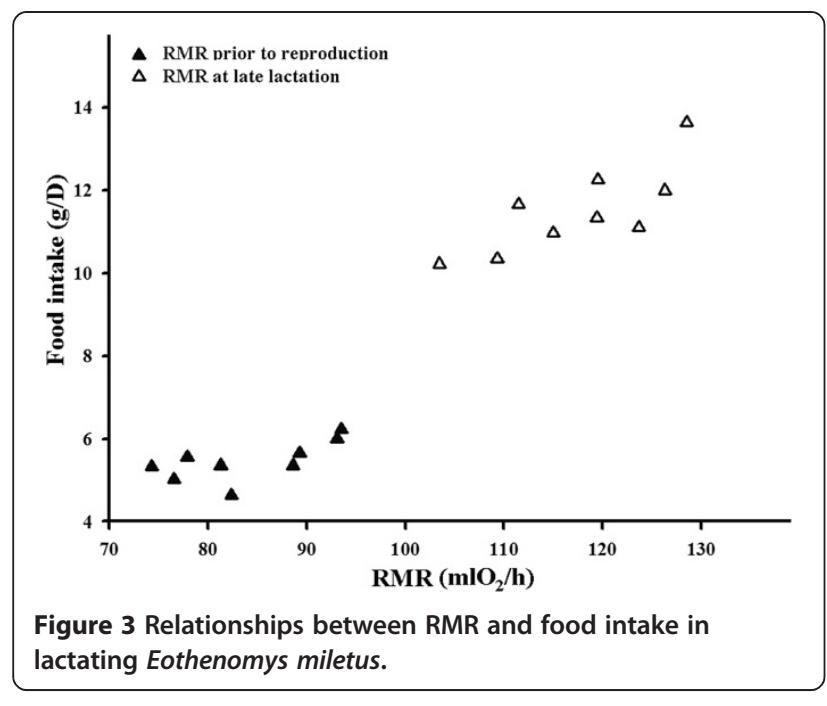

\section{Discussion}

Body mass, food intake, litter size, litter mass, and RMR In the present study, litter sizes ranged from 2 to 6 , but the majority litter size was 2 , and lactation period was 22 days, similar to our previous study (Zhu et al. 2013b). It showed that changes of RMR may affect the reproduction of female animals (Thompson and Nicoll 1986). In our study, RMR increased significantly (39.62\%) on day 22 of lactation compared with that prior to reproduction, and food intake increased $110.83 \%$ at the late lactation. Similar results were found in Mus musculus (Speakman and McQueenie 1996) and Mesocricetus auratus (Garton et al. 1994). However, some studies showed that RMR did not increase significantly during lactation, such as in Phodopus sungorus (Weiner 1987). Therefore, variation of RMR during lactation showed species-specific difference.

Variability of RMR was influenced by environmental variations (temperature, photoperiod, the quantity and quality of food), body mass, and reproductive status in mammals (Speakman 2007, 2008). In the present study, body mass was positively correlated with Pri RMR and LL RMR in lactating E. miletus, but no relationships were found between Pri RMR and litter size and mass of early lactation, and litter size and mass of late lactation, indicating that Pri RMR had no relation to reproductive output, which did not support the hypothesis that animals with higher Pri RMR often had larger reproductive output (Zhao et al. 2010c). However, LL RMR was positively correlated with litter size and mass of early lactation, litter size and mass, and food intake of late lactation. Most mammals, especially rodents, mainly increase food intake to compensate for energy expenditure, suggesting that higher RMR during late lactation is beneficial to maintain the digestive system, enhance the digestion and absorption capacity, and devote more energy for reproductive output (Thompson 1992).

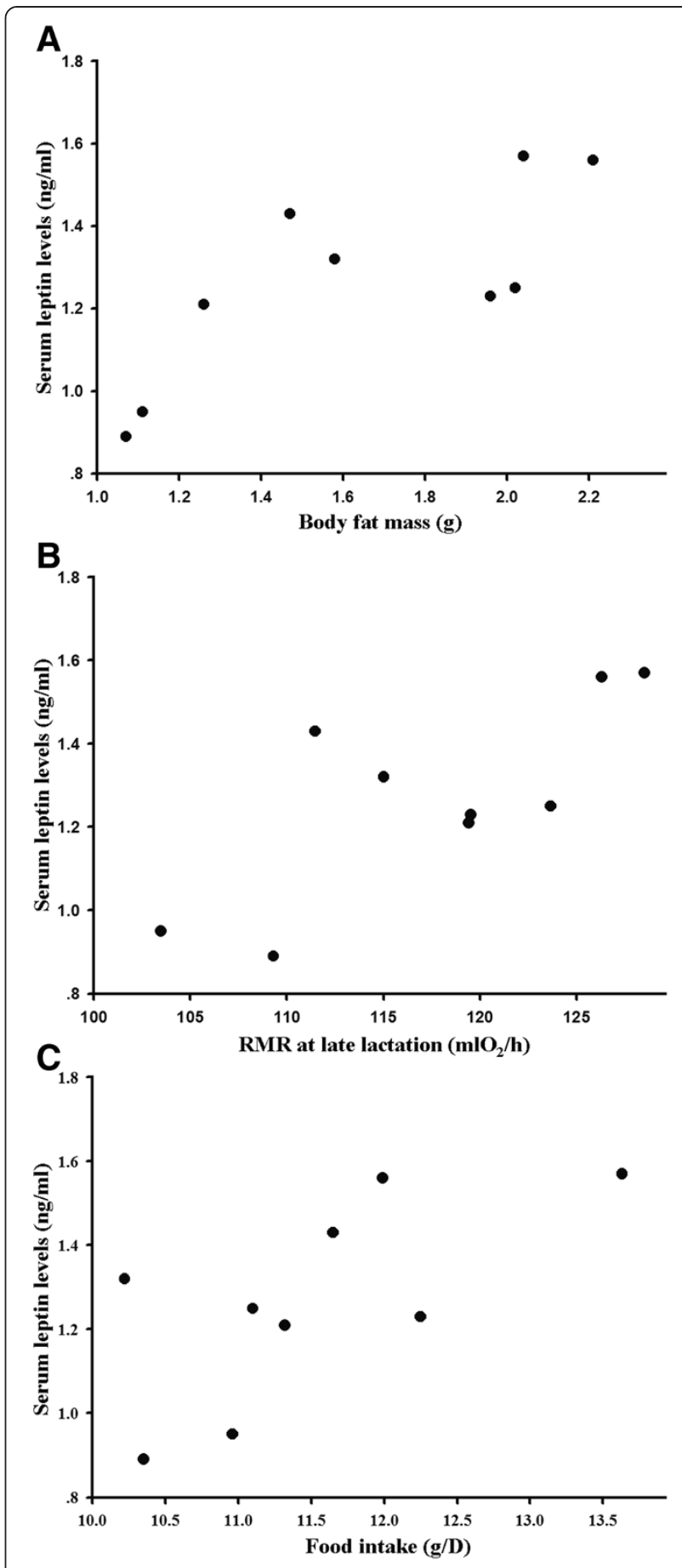

Figure 4 Serum leptin levels and body fat mass, LL RMR, food intake in lactating Eothenomys miletus. (A) Serum leptin levels vs. body fat mass. (B) Serum leptin levels vs. LL RMR. (C) Serum leptin levels vs. food intake.

\section{Serum leptin levels and RMR}

Leptin plays an important role in regulation of body mass (Abelenda et al. 2003). Circulating leptin concentrations reflect levels of body adiposity as referred in the 'adipostat hypothesis' (Tups et al. 2004). In general, 

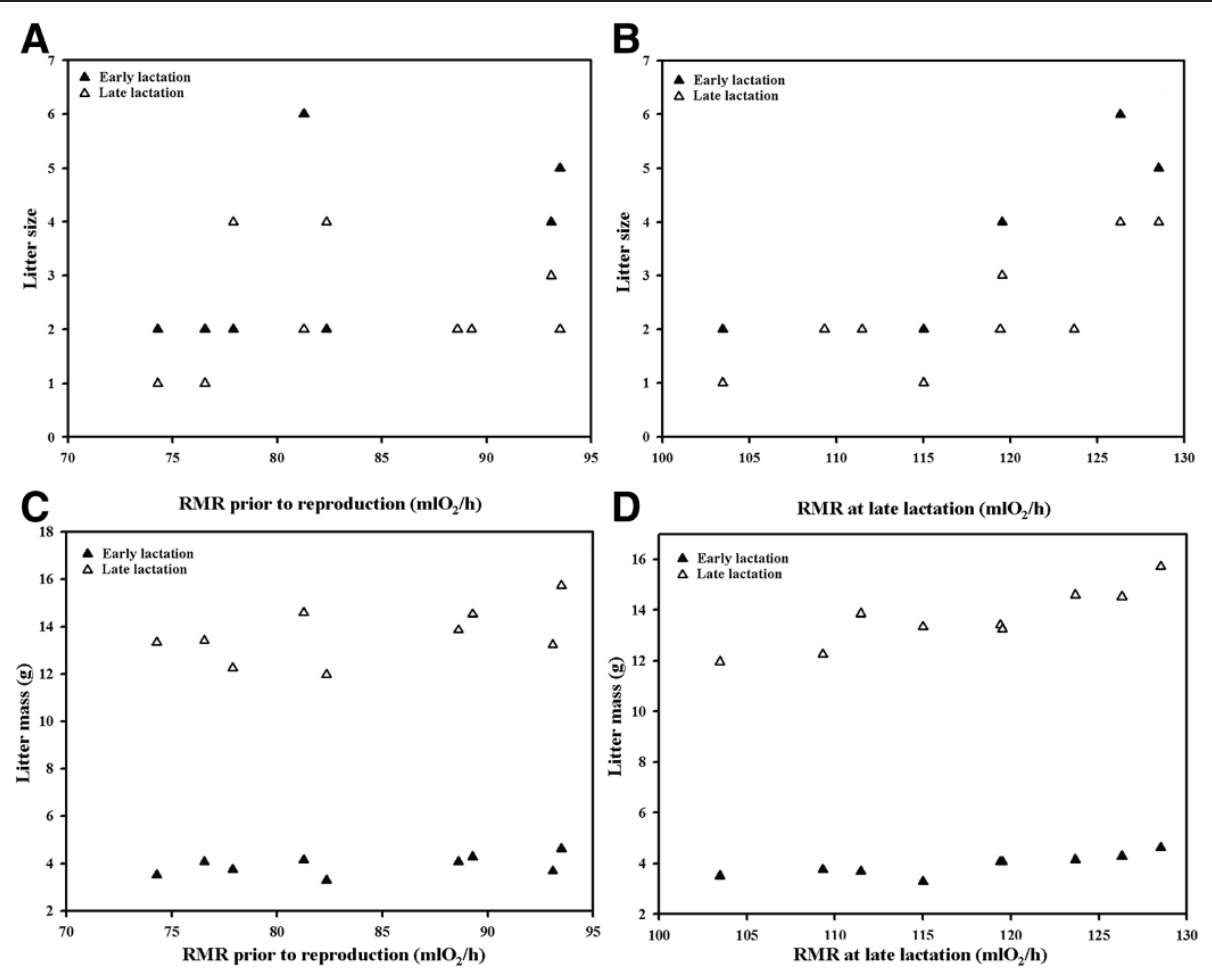

Figure 5 Litter size and RMR, and litter mass and RMR in Eothenomys miletus. (A) Litter size vs. RMR prior to reproduction. (B) Litter size vs. RMR at late lactation. (C) Litter mass vs. RMR prior to reproduction. (D) Litter mass vs. RMR at late lactation.

previous studies showed that serum leptin levels were positive correlated with body fat mass in small mammals, such as Meriones unguiculatus (Li and Wang 2005). In the present study, correlation analysis showed that serum leptin levels were positively correlated with body fat mass in lactating E. miletus, indicating that leptin may act as 'adiposity indicator' (Zhao and Wang 2006). To regulate body mass, leptin influences energy

Table 2 Relationships between RMR and organs in lactating Eothenomys miletus

\begin{tabular}{lll}
\hline Parameters & $\mathbf{r}$ & $\mathbf{P}$ \\
\hline Carcass & 0.532 & $<0.05$ \\
Heart & 0.756 & $<0.01$ \\
Liver & 0.784 & $<0.01$ \\
Lungs & 0.498 & $<0.05$ \\
Spleen & 0.635 & $<0.05$ \\
Kidneys & 0.701 & $<0.01$ \\
Brown adipose tissue (BAT) & 0.796 & $<0.01$ \\
Mammary gland & 0.832 & $<0.01$ \\
Stomach & 0.896 & $<0.01$ \\
Small intestine & 0.923 & $<0.01$ \\
Large intestine & 0.901 & $<0.01$ \\
Cecum & 0.879 & $<0.01$ \\
\hline
\end{tabular}

The data are the coefficient of correlation between the variables and RMR. balance via its effects on both food intake and energy expenditure (Concannon et al. 2001). In the current study, it showed that serum leptin levels were positively correlated with LL RMR and food intake in the late lactation, indicating that leptin was potentially involved in energy balance in lactating E. miletus. The present study supports our hypothesis that changes of body mass and thermogenic capacity might be mediated by leptin during lactation.

\section{Visceral organs, gastrointestinal tract, and RMR}

It has been confirmed that variation of RMR in lactation may be associated with reproductive output, which was mainly affected by morphological changes of organs (Poppitt et al. 1993). In the present study, LL RMR was positively correlated with carcass and weight of visceral organs and gastrointestinal tract, consistent with previous studies on Lasiopodomys brandtii (Zhao and Wang 2007). LL RMR was positively correlated with mammary gland, indicating that E. miletus during lactation need to increase the energy requirement for maintenance of mammary gland and finally to produce more milk (Rogowitz 1998). Furthermore, correlation analysis showed that RMR was more related to gastrointestinal tracts than the visceral organs. A similar result was also found in KM mice (Zhao et al. 2009). However, the opposite result was found in MF1 mice (Johnson et al. 2001). These results 
suggested that the relationships between RMR and visceral organs and gastrointestinal tract varied during lactation at interspecific levels. Our results supported the hypothesis that limitations of SusEI were centrally driven by the capacity of the gastrointestinal tract to process ingested food (Johnson et al. 2001).

\section{Conclusions}

In summary, RMR increased significantly at late lactation than prior to reproduction. There was no significant correlation between RMR prior to reproduction and reproductive output. However, RMR at late lactation was positively correlated with body mass, food intake, litter size and mass, and weight of visceral organs and gastrointestinal tract, supporting the hypothesis that higher RMR during late lactation is beneficial to enhance digestion and absorption capacity in the digestive system to increase energy intake for reproductive output. Moreover, leptin may be involved in body mass regulation during lactation in E. miletus.

\section{Abbreviations}

BAT: brown adipose tissue; LL RMR: RMR at late lactation; Pri RMR: RMR prior to reproduction; RMR: resting metabolic rate.

\section{Competing interests}

The authors declare that they have no competing interests.

\section{Authors' contributions}

Z-kW carried out the studies of body mass, RMR, and food intake and drafted the manuscript. W-IZ conceived the study and participated in its design and coordination. Both authors read and approved the final manuscript.

\section{Authors' information}

$\mathrm{W}-\mathrm{IZ}$ and $\mathrm{Z}-\mathrm{kW}$ are mainly engaged in the research of animal physiology and ecology.

\section{Acknowledgments}

This research was financially supported by the National Science Foundation of China (No. 31260097) and Project of Basilic Research of Yunnan Province (No. 2011FZ082). We wish to thank Prof. Burkart Engesser at Historisches Museum Basel, Switzerland for correcting the English usage in the draft. The two anonymous reviewers and the editor of the journal are also acknowledged for their valuable comments.

Received: 30 December 2013 Accepted: 4 July 2014

Published: 22 July 2014

\section{References}

Aoki N, Kawamura M, Matsuda T (1999) Lactation-dependent down regulation of leptin production in mouse mammary gland. BBA 1427:298-306

Abelenda M, Ledesma A, Rial E, Puerta M (2003) Leptin administration to coldacclimated rats reduces both food intake and brown adipose tissue thermogenesis. J Therm Biol 28:525-530

Concannon P, Levac K, Rawson R, Tennant B, Benadoun A (2001) Seasonal changes in serum leptin, food intake, and body weight in photo entrained woodchucks. Am J Physiol 281:951-959

Friedman JM, Halaas JL (1998) Leptin and the regulation of body weight in mammals. Nature 395:763-770

Garton DW, Hsu MJ, Harder JD (1994) Environmental temperature and metabolic rates during gestation and lactation in golden-hamsters (Mesocricetus auratus). Physiol Zool 67:497-514

Harvey PH, Pagel MD, Rees JA (1991) Mammalian metabolism and life histories. Am Nat 137:556-566
Hill RW (1972) Determination of oxygen consumption by use of the paramagnetic oxygen analyzer. J Appl Physiol 33:261-263

Hammond KA, Diamond J (1992) An experimental test for a ceiling on sustained metabolic-rate in lactating mice. Physiol Zool 65:952-977

Johnson MS, Thomson SC, Speakman JR (2001) Limits to sustained energy intake. II. Inter-relationship between resting metabolic rate, life-history traits and morphology in Mus musculus. J Exp Biol 204:1937-1946

Li XS, Wang DH (2005) Seasonal adjustments in body mass and thermogenesis in Mongolian gerbils (Meriones unguiculatus): the roles of short photoperiod and cold. J Comp Physiol 175:593-600

Poppitt SD, Speakman JR, Racey PA (1993) The energetics of reproduction in the common shrew (Sorex areneusi), a comparison of indirect calorimetry and the doubly labeled water technique. Physiol Zool 66:964-982

Rogowitz GL (1998) Limits to milk flow and energy allocation during lactation in the hispid cotton rat (Sigmodon hispidus). Physiol Zool 71:312-320

Seeber RM, Smith JT, Waddell BJ (2002) Plasma leptin-binding activity and hypothalamic leptin receptor expression during pregnancy and lactation in the rat. Biol Reprod 66:1762-1767

Selman C, Lumsden S, Bunger L, Hill WG, Speakman JR (2001) Resting metabolic rate and morphology in mice (Mus musculus) selected for high and low food intake. J Exp Biol 204:777-784

Speakman JR, McQueenie J (1996) Limits to sustained metabolic rate: the link between food intake, basal metabolic rate and morphology in reproducing mice, Mus musculus. Physiol Zool 69:746-769

Speakman JR (2000) The cost of living: field metabolic rates of small mammals. Adv Ecol Res 30:177-297

Speakman JR, Król E (2005) Limits to sustained energy intake. IX. A review of hypotheses. J Comp Physiol 175:375-394

Speakman JR (2007) The energy cost of reproduction in small rodents. Acta Theriol Sin 27:1-13

Speakman JR (2008) The physiological cost of reproduction in small mammals. Phil trans Roy Soc 363:375-398

Speakman JR, Król E (2010) Maximal heat dissipation capacity and hyperthermia risk: neglected key factors in the ecology of endotherms. J Anim Ecol 79:726-746

Thompson SD, Nicoll ME (1986) Basal metabolic rate and energetics of reproduction in therian mammals. Nature 321:690-693

Thompson SD (1992) Gestation and lactation in small mammals: basal metabolic rate and the limits to energy use. In: Tomasi TE, Horton TH (eds) Mammalian energetics: interdisciplinary views of metabolism and reproduction. Cornell University Press, Ithaca, New York, pp 213-259

Tups A, Ellis C, Moar KM, Logie TJ, Adam CL, Mercer JG, Klingenspor M (2004) Photoperiodic regulation of leptin sensitivity in the Siberian hamster, Phodopus sungorus, is reflected in arcuate nucleus SOCS-3 (suppressor of cytokine signaling) gene expression. J Endocrinol 145:1185-1193

Weiner J (1987) Limits to energy budget and tactics in energy investments during reproduction in the Djungarian hamster (Phodopus sungorus sungorus Pallas 1770). Symp Zool Soc Land 57:167-187

Zhang XY, Wang DH (2007) Thermogenesis, food intake and serum leptin in cold-exposed lactating Brandt's voles Lasiopodomys brandtii. J Exp Biol 210:512-521

Zhao ZJ, Wang DH (2006) Effects of photoperiod on energy budgets and thermogenesis in Mongolian gerbils (Meriones unguiculatus). J Therm Biol 31:323-331

Zhao ZJ, Wang DH (2007) Effects of diet quality on energy budgets and thermogenesis in Brandt's voles. Comp Biochem Physiol 148:168-177

Zhao ZJ, Cao J, Wang GY, Ma F, Meng XL (2009) Effect of random food deprivation and re-feeding on energy metabolism and behavior in mice. Acta Theriol Sin 29:277-285

Zhao ZJ (2010) Energy budget during lactation in striped hamsters at different ambient temperatures. J Exp Biol 214:988-995

Zhao ZJ, Chi QS, Cao J (2010a) Milk energy output during peak lactation in shaved Swiss mice. Physiol Behav 101:59-66

Zhao ZJ, Chi QS, Cao J (2010b) Limits to sustainable energy budget during lactation in the striped hamster (Cricetulus barabensis) raising litters of different size. Zool 113:235-242

Zhao Z, Cao J, Li LS (2010c) Relationships between basal metabolic rate and reproductive output and visceral organs in lactating mice. Chinese J Zool 45:39-45

Zhu WL, Wang B, Cai JH, Lian X, Wang ZK (2011a) Thermogenesis, energy intake and serum leptin in Apodemus chevrieri in Hengduan Mountains region during cold acclimation. J Therm Biol 36:181-186 
Zhu WL, Cai JH, Lian X, Wang ZK (2011b) Effects of photoperiod on energy intake, thermogenesis and body mass in Eothenomys miletus in Hengduan Mountain region. J Therm Biol 36:380-385

Zhu WL, Yang SC, Wang ZK (2012) Adaptive characters of energy metabolism, thermogenesis and body mass in Eothenomys miletus during cold exposure and rewarming. Anim Biol 62:263-276

Zhu WL, Yang G, Zhang L, Wang ZK (2013a) Effects of photoperiod and temperature on the body mass, thermogenesis, and serum leptin levels of Apodemus draco (Rodentia: Muridae) in the Hengduan Mountain region, China. Zool Stud 52:20

Zhu WL, Mu Y, Zhan L, Wang ZK (2013b) Effect of temperature on body temperature and resting metabolic rate in pups of Eothenomys miletus. J Stress Physiol Biochem 9:148-156

\section{doi:10.1186/s40555-014-0041-3}

Cite this article as: Zhu and Wang: Resting metabolic rate and energetics of reproduction in lactating Eothenomys miletus from Hengduan mountain region. Zoological Studies 2014 53:41.

\section{Submit your manuscript to a SpringerOpen ${ }^{\circ}$ journal and benefit from:}

- Convenient online submission

- Rigorous peer review

- Immediate publication on acceptance

- Open access: articles freely available online

- High visibility within the field

- Retaining the copyright to your article 